\title{
Short-term Effects of Fumigation with Gaseous Methanol on Photosynthesis in Horticultural Plants
}

\author{
Francesco Loreto, Domenico Tricoli, and Mauro Centritto \\ CNR, Istituto di Biochimica ed Ecofisiologia Vegetali, Via Salaria Km. 29,300-00016 Monterotondo \\ Scalo (Roma) Italy
}

\author{
Arturo Alvino and Sebastiano Delfine \\ Università del Molise, Dipartimento SAVA, 86100 Campobasso, Italy
}

AdDitional INDEX WORDS. electron transport rate, gas exchange, photosynthesis, photorespiration, stomatal conductance

\begin{abstract}
Short-term fumigation with $1 \%$ methanol in air was carried out to investigate effects on the photosynthetic apparatus of horticultural species characterized by leaves with different stomatal distribution. Methanol decreased the photosynthetic capacity of all species. The hypostomatous cherry (Prunus avium L.) was the most sensitive species. Between the two amphistomatous species, the effect was smaller in pepper (Capsicum annuum L. var. annuum) than in melon (Cucumis melo L.). A 4-minute fumigation caused a stronger inhibition of photosynthesis than a 90-second fumigation. The time course of the inhibition of the photosynthetic electron transport following a methanol fumigation of cherry leaves suggests that methanol starts inhibiting photosynthesis and photorespiration after $\approx 60$ seconds and that the effect is complete after 180 seconds. This inhibition is not permanent, however, since gas-exchange properties recovered within 24 hours. Methanol vapor effects were greatest when leaves were fumigated on the surfaces with stomata. However, fumigation with methanol does not affect stomatal conductance. Therefore, inhibition of photosynthesis following methanol fumigation can be attributed to a temporary inhibition of biochemical reactions.
\end{abstract}

Foliar sprays of methanol solutions were reported to increase the yield of crops (Nonomura and Benson, 1992). Other experiments confirmed (Valenzuela et al., 1994) or disproved (Albrecht et al., 1995; Faver and Gerik, 1996; McGiffen et al., 1995; van Iersel et al., 1995) that methanol has a beneficial effect on crop yield. Yield improvement was especially noticed in $\mathrm{C}_{3}$ plants growing in arid, high sunlight environments but was not observed in $\mathrm{C}_{4}$ plants (Nonomura and Benson, 1992). Apparently, the leaf turgor of methanol-sprayed leaves was higher than in nonstressed controls. This led to speculation that methanol spraying increased stomatal conductance, in turn resulting in improved carbon acquisition (Nonomura and Benson, 1992). However, Nonomura and Benson (1992) did not report any direct measurements of $\mathrm{CO}_{2}$ and $\mathrm{H}_{2} \mathrm{O}$ exchange between leaves and air. More recently, methanol was found to improve photosynthesis and stomatal conductance but not growth of cotton (Faver and Gerik, 1996) and was ineffective on sour orange (Idso et al., 1995) and on fescue (Poskuta et al., 1995). A first goal of our work was to understand how stomata are affected by methanol. We investigated the effect of methanol on horticultural plants with different stomatal distribution as estimated by exchange of water vapor in a gas exchange cuvette. We used one hypostomatous species (cherry) and two species amphistomatous but with stomata prevalently distributed on the abaxial (pepper) and on the adaxial (melon) leaf surface, respectively. This approach also allowed us to investigate if methanol penetration occurred through stomata and/or cuticle and if the methanol effect on photosynthesis was mediated by changes of stomatal opening.

Nonomura and Benson (1992) also speculated that an inhibition of photorespiration could improve carbon fixation of $\mathrm{C}_{3}$ plants exposed to methanol sprays. This would have explained why the effect was not observed in $\mathrm{C}_{4}$ plants. Gas exchange-based

Received for publication 15 June 1998. Accepted for publication 8 Mar. 1999. The cost of publishing this paper was defrayed in part by the payment of page charges. Under postal regulations, this paper therefore must be hereby marked advertisement solely to indicate this fact. reports show that methanol does not affect the $\mathrm{CO}_{2}$ compensation point (Poskuta et al., 1995, van Iersel et al., 1995), an indication that photorespiration is not preferentially suppressed by methanol. Simultaneous measurements of gas exchange and chlorophyll fluorescence, allow one to partition the electron transport rate between photosynthesis and photorespiration (Di Marco et al., 1993). We used this approach to accomplish our second goal, that is, to understand if methanol vapor rapidly affected biochemistry of photosynthesis and selectively inhibited photorespiration.

It is possible that the different results obtained with methanol sprays reflect differences in the exposure and acquisition of methanol by the leaf. Under field conditions these cannot be controlled accurately. For instance, acquisition may be inefficient because of temperature-dependent volatilization of the methanol solution. The third goal of this study was to eliminate these uncertainties while investigating methanol effects on stomata and biochemistry. We used gaseous methanol in a totally controlled environment. This eliminated the variability of environmental factors and allowed us to study a direct effect of methanol vapor rather than an effect that may depend on the environment-controlled evolution of methanol vapor from sprays.

\section{Materials and Methods}

Seedlings of pepper (Capsicum annuum 'Quadrato d'Asti') and melon (Cucumis melo 'Supermarket') were grown for $\approx 2$ months in $3-\mathrm{dm}^{3}$ pots. Two-year-old cherry (Prunus avium 'Gisella') trees were grown in $15-\mathrm{dm}^{3}$ pots. Soil was a mixture of 1 sand : 1 peat : 1 lime. Pots were regularly watered to soil saturation and weekly fertilized according to the Ingestad and Agren (1992) method. Plants were grown under conditions typical of summer in the experimental field of IBEV (Rome, Italy $41^{\circ}$ $50^{\prime}$ latitude). Daily light intensity and air temperature varied between 700 to $2000 \mu \mathrm{mol} \cdot \mathrm{m}^{-2} \cdot \mathrm{s}^{-1}$ and 25 to $37^{\circ} \mathrm{C}$, respectively, depending of sky cloudiness.

The experiments were conducted on the last fully expanded leaf. The leaf was enclosed in a gas exchange cuvette with $4.9-\mathrm{cm}^{2}$ 
windows to allow illumination and fluorescence detection. The leaf was maintained at a temperature of $30^{\circ} \mathrm{C}$ while exposed to a light intensity of $1000 \mu \mathrm{mol} \cdot \mathrm{m}^{-2} \cdot \mathrm{s}^{-1}$ and to ambient $\mathrm{O}_{2}$ and $\mathrm{CO}_{2}$ partial pressures (20 and $350 \mu$ bar, respectively). Under these conditions the vapor pressure difference between leaf and air was always maintained below $20 \mathrm{mbar}^{\mathrm{bar}} \mathrm{b}^{-1}$ and leaf stomatal opening was near maximum. Photosynthesis and stomatal conductance were calculated on a projected area basis from gas-exchange measurements. Other details of the fluorescence and gas-exchange system and measurements are reported in Loreto et al. (1994) and Delfine et al. (1998).

Treated leaves were exposed to air containing $\approx 1 \%$ methanol after reaching steady-state photosynthesis and stomatal conductance. The methanol concentration was achieved by bubbling the compressed air in a solution of methanol (30\% in water) before entering the cuvette. The glass tube containing the methanol solution was placed in a water bath refrigerated at $9{ }^{\circ} \mathrm{C}$ to allow controlled volatilization of the compound. From the volume of methanol solution and its constants, we calculated that at $9{ }^{\circ} \mathrm{C}$ the vapor pressure of methanol in the gas phase was $8.38 \mathrm{~mm}$, or $1.1 \%$ of the total pressure.

Fumigation was carried out for $90 \mathrm{~s}$ or $4 \mathrm{~min}$, by operating two three-way electrovalves, which allowed the air flux to alternatively include or bypass the methanol solution. The cuvette allowed to separate the flux of air flowing over the two leaf surfaces. Methanol was added to the air flowing over both leaf surfaces during the 90-s treatment. During the 4-min treatment, methanol was added either to the air flowing over both leaf surfaces or, alternatively, to the air flowing over either the abaxial or adaxial surface only. After each experiment, leaves were returned to methanol-free air and gas exchange was monitored after $5 \mathrm{~min}$. This was the minimum time necessary to stabilize the reading of the infrared gas analyzer (LI-6262, LI-COR, Lincoln, Neb.). We did not attempt to follow gas exchange during fumigation because the effect of methanol on the infrared gas analyzer performance is unknown. Moreover, the instantaneous change of pressure caused by inserting the glass tube containing methanol in the flow path made it impossible to accurately measure gas exchange over periods as short as 1 min. During fumigation, however, we followed chlorophyll fluorescence, which gave us an instantaneous estimation of the photosynthetic electron transport (Loreto et al., 1994). By comparing gas exchange and fluorescence measurements we were also able to derive the velocity of carboxylation $\left(\mathrm{v}_{\mathrm{c}}\right)$ and the velocity of oxygenation $\left(\mathrm{v}_{\mathrm{o}}\right)$ of ribulose-1,5-bisposphate carboxylase/oxygenase, as shown by $\mathrm{Di}$ Marco et al. (1993).

All experiments were conducted using four leaves of different plants and the average \pm standard error is reported. The time course of electron transport inhibition measured by fluorescence is shown for only one leaf for clarity.

\section{Results}

Photosynthesis and stomatal conductance of hypostomatous cherry leaves occurred only through the abaxial surface (Fig. 1). On the other hand, the leaves of the two herbaceous species could have measurable gas exchange through both surfaces. As shown by the different distribution of stomatal conductance between abaxial and adaxial leaf surfaces, however, pepper is dominantly hypostomatous, while melon is dominantly epistomatous.

Photosynthesis of cherry and melon leaves exposed to methanol for $90 \mathrm{~s}$ was reduced with respect to controls (Fig. 2a and b). However, photosynthesis of pepper leaves was not significantly affected by this $90-\mathrm{s}$ treatment.

Fumigation of both leaf surfaces with methanol for $4 \mathrm{~min}$ resulted in a dramatic decline of leaf photosynthesis in all species. Cherry leaves showed a positive $\mathrm{CO}_{2}$ release whereas photosynthesis of melon leaves was near the compensation point. Pepper leaf photosynthesis was less affected by the 4-min treatment than photosynthesis of the two other species.

When fumigation was limited to the abaxial leaf surface, photosynthesis of cherry leaves was again totally inhibited. Abaxial fumigation reduced photosynthesis of melon and pepper leaves less than fumigation of both leaf surfaces. Fumigation of the adaxial leaf surface only did not cause any change in cherry leaf photosynthesis. This treatment reduced photosynthesis more than the treatment to the abaxial surface but less than the treatment to both surfaces of melon leaves. In pepper leaves, photosynthesis inhibition after methanol fumigation of the adaxial surface was lower than that observed when fumigating the abaxial or both surfaces (Fig. 2).

Stomatal conductance of leaves was not affected by fumigation with methanol irrespective of plant species, duration and localization of the treatment. Even the treatment that most effectively inhibited photosynthesis (a 4-min fumigation of the entire leaf) did not cause stomatal closure (Table 1). After $24 \mathrm{~h}$, photosynthesis and stomatal conductance of the methanol-treated leaves was comparable to that observed before fumigation (not shown).

We followed the time course of the methanol effect on the photosynthetic apparatus by monitoring the chlorophyll fluores-
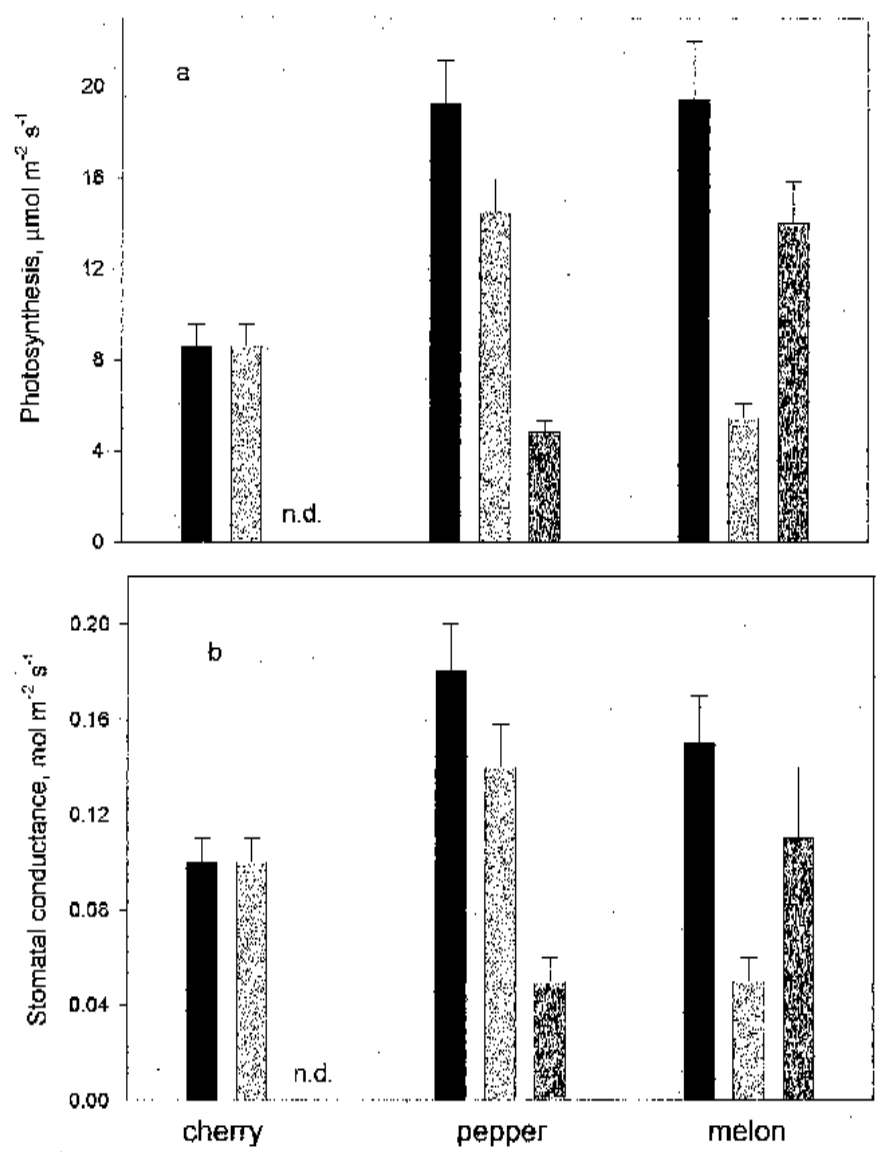

Fig. 1. Photosynthesis (a) and stomatal conductance (b) of nonfumigated leaves of cherry, pepper and melon. Total gas exchanges (black) are compared with the gas exchange of the abaxial (light gray) and adaxial (dark gray) leaf surfaces. n.d. $=$ not detectable. Bars are means of 4 measurements (one leaf on 4 different plants). Error bars represent the standard error. 
cence and by calculating the photosynthetic electron transport in cherry leaves. We found that electron transport was generally not affected for the first $60 \mathrm{~s}$, then dropped rapidly. Electron transport was almost completely suppressed after $180 \mathrm{~s}$ and did not recover 5 min after switching to methanol-free air (Fig. 3b). A similar effect was observed when fumigating the abaxial leaf surface only, while fumigation of the adaxial surface did not change the electron transport rate.

The ratio between velocity of oxygenation and of carboxyla-

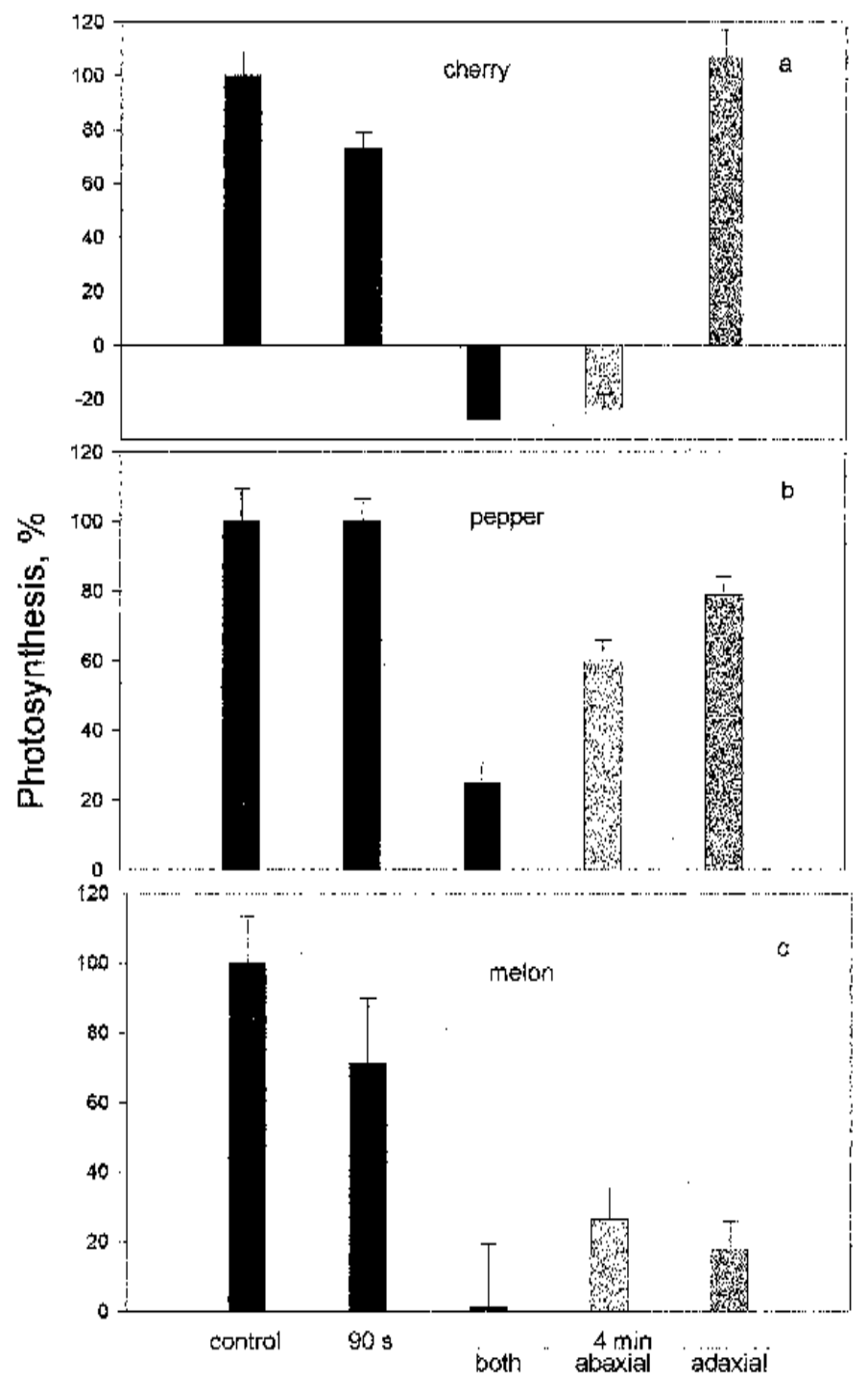

Fig. 2. Inhibition of photosynthesis caused by a 90 -s or 4-min fumigation with $1 \%$ methanol in air over cherry (a), pepper (b), and melon (c) leaves. Controls are the same leaves before fumigation. The 4-min fumigation was applied to the entire leaf (black) or alternatively to the abaxial (light gray) and adaxial (dark gray) leaf surface. Bars are means of four measurements (one leaf on four different plants). Error bars represent the standard error.

Table 1. Stomatal conductance of cherry, pepper and melon leaves before and after a 4-min fumigation with $1 \%$ methanol. Means \pm standard errors $(n=4)$ are shown.

\begin{tabular}{lccc}
\hline \hline & \multicolumn{3}{c}{ Stomatal conductance $\left(\mathrm{mol} \cdot \mathrm{m}^{-2} \cdot \mathrm{s}^{-1}\right)$} \\
\cline { 2 - 4 } Condition & Cherry & Pepper & Melon \\
\hline Before fumigation & $0.10 \pm 0.01$ & $0.18 \pm 0.02$ & $0.15 \pm 0.02$ \\
After a 4-min fumigation & $0.09 \pm 0.02$ & $0.20 \pm 0.03$ & $0.15 \pm 0.03$ \\
\hline
\end{tabular}

tion was calculated before and after the fumigation of both surfaces in cherry leaves. A significant increase $(\approx 30 \%)$ of the ratio was noticed after a 4-min methanol fumigation (Fig. 3a).

\section{Discussion}

We observed a negative effect of the methanol fumigation on the photosynthesis of all three species. The effect appeared to be associated with the stomatal distribution in leaves. No effect was observed when we fumigated the adaxial surface of the hypostomatous cherry leaves. Photosynthesis reduction was partially recovered when only one surface of the amphistomatous leaves of pepper and melon was fumigated with respect to fumigation of both surfaces. The recovery was stronger when the fumigated leaf surface contained fewer stomata, i.e. the abaxial surface in pepper and the adaxial surface in melon. These data indicate that methanol acquisition principally occurs through stomata openings. Acquisition by diffusion through the cuticle of the leaves cannot be discarded. However, as shown by the undetectable effect of fumigation of the adaxial surface of the hypostomatous cherry leaves, cuticle does not seem to contribute, at least over the short term, to methanol acquisition.

The inhibition of photosynthesis in methanol-treated leaves was greater in cherry leaves than in the leaves of the two herbaceous species. Pepper, in particular, showed a much lower inhibitory effect than the other species. Stomatal conductance was lower in cherry than in pepper and melon (Fig. 1) and was not affected by the methanol fumigation (Table 1). Thus, we infer that the acquisition of methanol by the leaf is poorly regulated by stomatal opening. The methanol-related inhibition of photosynthesis was small when the fumigation was limited to $90 \mathrm{~s}$ but

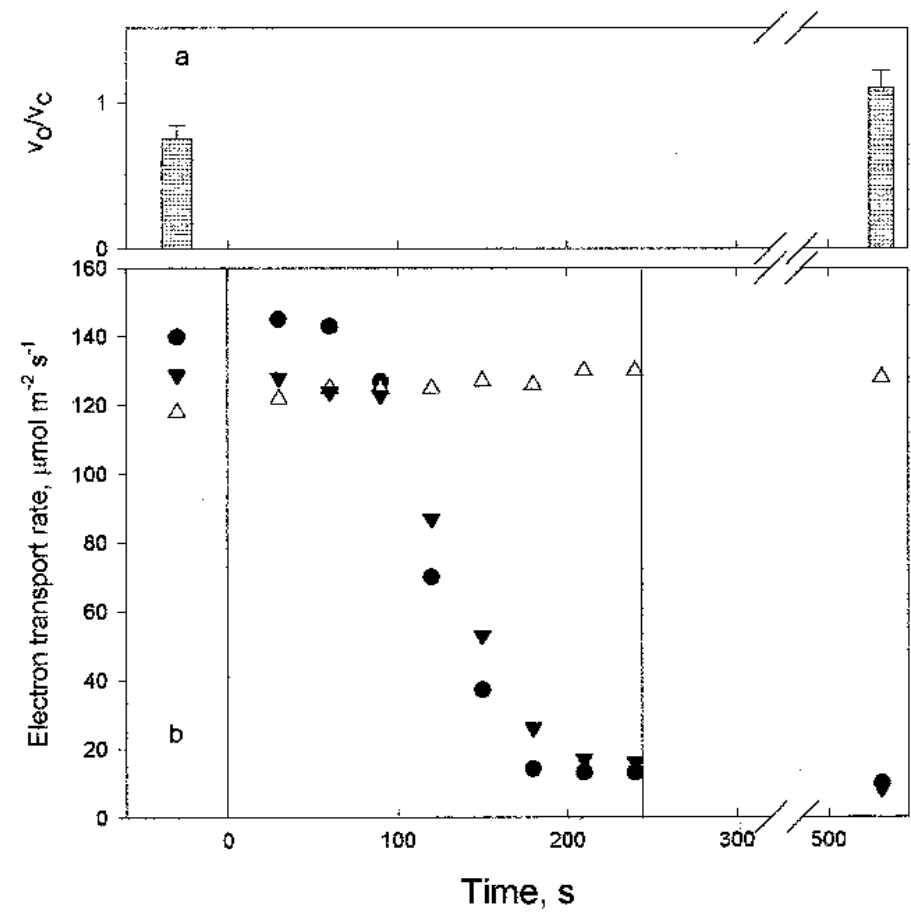

Fig. 3. Ratio between the velocity of oxygenation and of carboxylation $\left(\mathrm{v}_{\mathrm{o}} / \mathrm{v}_{\mathrm{c}}\right)$ before and after a 4-min fumigation with $1 \%$ methanol in air over cherry leaves (a). Bars are means of four measurements (one leaf on four different plants). Error bars represent the standard error. In the panel (b), the time course of the inhibition of the photosynthetic electron transport of a cherry leaf, measured through fluorescence, during a 4-min fumigation with $1 \%$ methanol in air, is shown. (-) Fumigation to the entire leaf. Triangles are referred to fumigation of the abaxial $(\boldsymbol{\nabla})$ and adaxial $(\Delta)$ leaf surface. 
became greater when fumigation was carried out for $4 \mathrm{~min}$. In both cases, cherry was the most sensitive and pepper the less sensitive among the tested species. Since species sensitivity was not associated with specific stomatal conductance, the different methanol effect suggests a different sensitivity at the biochemical level among species.

We were surprised to observe an inhibition of photosynthesis since an influential early report indicated that methanol sprays induced higher crop yields, supposedly by improving stomatal opening and therefore carbon acquisition (Nonomura and Benson, 1992). We did not observe any change of stomatal opening during the treatment or after a $24 \mathrm{~h}$ recovery. Therefore, a) it can be excluded that the observed photosynthesis inhibition is caused by stomatal closure, and b) an increased turgidity, as proposed to be caused by stomatal opening, is unlikely to be responsible for the previously observed improvement of crop yields following methanol sprays.

It should be noted that Nonomura and Benson (1992) observed recurrent toxicity of the methanol treatment and methanol-related leaf injuries when spraying water solutions with $10 \%$ to $50 \%$ methanol. Albrecht et al. (1995) reported leaf toxicity at even higher concentrations of methanol (above $60 \%$ of the aqueous solution). We applied a concentration of methanol (1\% in air), which was by far lower than the concentration that should volatilize when spraying at moderate to high air temperatures these concentrations of methanol in water solution. Therefore, we did not expect to injure the leaves. Any toxicity of the treatment was apparently totally reversible since after $24 \mathrm{~h}$ photosynthesis of fumigated leaves completely recovered. This was true whether the exposure was limited to $90 \mathrm{~s}$ or extended to $4 \mathrm{~min}$.

To characterize the effect of methanol on the photosynthetic apparatus we followed the electron transport rate during a 4-min fumigation of cherry leaves. The electron transport measured through fluorescence, is representative of photosynthesis and photorespiration (Loreto et al., 1994). The ratio between the velocity of oxygenation $\left(\mathrm{v}_{\mathrm{o}}\right)$ and carboxylation $\left(\mathrm{v}_{\mathrm{c}}\right)$ can be derived by coupled measurements of fluorescence and gas-exchange (Di Marco et al., 1993). Since gas-exchange measurements were not monitored during fumigation, the $\mathrm{v}_{\mathrm{o}} / \mathrm{v}_{\mathrm{c}}$ ratio could be calculated only before and after fumigation. There was about a $30 \%$ increase of the $\mathrm{v}_{\mathrm{o}} / \mathrm{v}_{\mathrm{c}}$ ratio after the methanol fumigation with respect to before fumigation (Fig. 3a). However, both photosynthesis and photorespiration were extremely low in cherry leaves after a 4min fumigation (Fig. 3b). This casts a doubt on whether the increase of the $\mathrm{v}_{\mathrm{o}} / \mathrm{v}_{\mathrm{c}}$ ratio reflects a lower sensitivity to methanol of the metabolites of the photorespiratory pathway compared with the sensitivity of the carbon fixation metabolites. In absence of more compelling evidence, we suggest that methanol inhibits a common precursor of photosynthesis and photorespiration. It was proposed that photorespiratory intermediates are used to detoxify methanol and that this could in turn favor carbon fixation over photorespiration (Nonomura and Benson, 1992). We can reject this hypothesis on the basis of our results, since the increase in the $\mathrm{v}_{\mathrm{o}} / \mathrm{v}_{\mathrm{c}}$ ratio after fumigation indicate that photorespiration may in fact be sustained, perhaps because it feeds electrons to scavenge methanol toxic by-products.

There was no effect on the electron transport rate when fumigation lasted less than $60 \mathrm{~s}$. Exposure to methanol for longer than 60 s resulted in a rapid inhibition of electron transport to over $95 \%$ of the prefumigation electron transport after $\approx 180 \mathrm{~s}$, which did not recover $5 \mathrm{~min}$ after switching off the methanol fumigation.
This was observed both when fumigating the entire leaf or only the abaxial surface of the hypostomatous cherry leaves. Methanol fumigation shorter than $60 \mathrm{~s}$ was probably not enough to let methanol pass through stomata and reach the chloroplasts where it was presumably toxic. Perhaps the previously observed positive effect of methanol on plant growth and yield was achieved only when the volatilization of sprayed methanol during field tests was so rapid that it was available to the leaf for very short periods. This may explain why a yield increase was observed mainly in arid environments and under semidesert conditions (Nonomura and Benson, 1992). However, our results offer no physiological explanation about the reason why methanol should improve plant yield. When the methanol uptake by the leaf occurs for longer than $60 \mathrm{~s}$, as for instance when volatilization is reduced by low temperatures, only a transitory toxic effect on the photosynthetic apparatus is expected.

In conclusion, our data showed that methanol is acquired through stomata but that stomata do not respond to methanol vapor. They also indicated that methanol acquisition occurs within minutes and revealed a short-term toxic effect of methanol on the biochemistry of photosynthesis.

\section{Literature Cited}

Albrecht, S.L., C.L. Douglas, E.L. Klepper, P.E. Rasmussen, R.W. Rickman, R.W. Smiley, D.W. Wysocki, D.E. Wilkins, and D.J. Wysocki. 1995. Effects of foliar methanol applications on crop yield. Crop Sci. 35:1642-1646.

Delfine S., A. Alvino, M. Zacchini, and F. Loreto. 1998. Consequences of salt stress on diffusive conductances, rubisco characteristics and anatomy of spinach leaves. Austral. J. Plant Physiol. 25:395-402.

Di Marco, G., M.A. Iannelli, and F. Loreto. 1993. Relationship between photosynthesis and photorespiration in field-grown wheat leaves. Photosynthetica 30:45-51.

Faver, K.L. and T.J. Gerik. 1996. Foliar-applied methanol effects on cotton (Gossypium hirsutum L.) gas exchange and growth. Field Crop Res. 47:227-234.

Idso, S.B., K.E. Idso, R.L. Garcia, B.A. Kimball, and J.K. Hoober. 1995. Effects of atmospheric $\mathrm{CO}_{2}$ enrichment and foliar methanol application on net photosynthesis of sour orange tree (Citrus aurantium; Rutaceae) leaves. Amer. J. Bot. 82:26-30.

Ingestad, T. and G.I. Agren. 1992. Theories and methods on plant nutrition and growth. Physiol. Plant. 84:177-184.

Loreto F., G. Di Marco, D. Tricoli, and T.D. Sharkey. 1994. Measurements of mesophyll conductance, photosynthetic electron transport and alternative electron sinks of field grown wheat leaves. Photosynth. Res. 41:397-403.

McGiffen, Jr., M.E., R.L. Green, J.A. Manthey, B.A. Faber, A.J. Downer, N.J. Sakovich, and J. Aguiar. 1995. Field tests of methanol as a crop yield enhancer. HortScience 30:1225-1228.

Nonomura, A.M. and A.A. Benson. 1992. The path of carbon in photosynthesis: Improved crop yields with methanol. Proc. Natl. Acad. Sci. USA 89:9794-9798.

Poskuta, J.W., E. Parys, E. Waclawczyk-Lach, and B. Wroblewska. 1995. Foliar sprays of methanol ineffective in activity of photosystem II, amount of photosynthetic pigments, $\mathrm{CO}_{2}$ exchange in light and in darkness and herbage yield of hexaploid and decaploid tall fescue genotypes. Acta Physiol. Plant. 17:91-93.

Valenzuela, H.R., O. Kawabata, and H. Yamamoto. 1994. Effect of methanol on biomass accumulation and photosynthetic efficiency of vegetable crops in Hawaii. HortScience 29:510.

Van Iersel, M.W., J.J. Heitholt, R. Wells, and D.M. Oosterhuis. 1995. Foliar methanol applications to cotton in the Southern United States: Leaf physiology, growth and yield components. Agron. J. 87:11571160 . 